\title{
EVALUACIÓN DEL RIESGO POR MOVIMIENTOS DE REMOCIÓN EN MASA ACTIVADOS POR PROCESOS DE PRECIPITACIÓN, ESTUDIO DE CASO VÍA CHAGUALÁ - QUINDÍO.
}

\author{
RISK EVALUATION FOR MASS REMOVAL MOVEMENTS ACTIVATED BY PRE- \\ CIPITATION PROCESSES, CASE STUDY AT CHAGUALÁ ROAD - QUINDÍO.
}

\author{
Cesar Augusto Rodríguez Mejía ${ }^{1}$, David Esteban Bustos Castelblanco ${ }^{2 *}$, \\ Alethia Cadena Acevedo ${ }^{3}$, Dany Mishell Salazar Henao ${ }^{4}$. \\ 1. Universidad del Quindío, e-mail: carodriguezm@uniquindio.edu.co \\ 2. Universidad del Quindío, e-mail: debustosc@uqvirtual.edu.co \\ 3. Universidad del Quindío, ale.cacevedo@hotmail.com \\ 4. Universidad del Quindío, danymishell.s.h@gmail.com
}

*Correspondencia del Autor: David Esteban Bustos Castelblanco. Dirección: Cll 5 No 16 - 11 Piso 2, Teléfono:

3004087226, Correo electrónico: debustoc@uqvirtual.edu.co

\section{RESUMEN}

En el presente documento se presenta la evaluación del riesgo por movimientos de remoción en masa activados por procesos de precipitación sobre la vía Chagualá del departamento del Quindío; para la cual se toma como lineamiento las metodologías desarrolladas por la Unidad Nacional para la Gestión del Riesgo y del Desastre (UNGRD) y el Servicio Geológico Colombiano, que consisten en la estimación de la amenaza por medio del análisis científico de los parámetros hidroclimatológicos, geológicos, físico, geométricos y mecánicos de la zona de estudio, así como la estimación del factor de riesgo, a través del método de Bishop simplificado; la determinación de vulnerabilidad se basa en matrices de impacto y por medio del cruce de información se evalúa el riesgo de deslizamiento por precipitación. Los resultados del estudio, demostraron que el valor y la variación de la intensidad de la lluvia en función del periodo de retorno es despreciable, comparada con la lámina de agua generada por la precipitación efectiva total anual, además el análisis de riesgo mostró un nivel alto ante un movimiento de remoción en masa considerando como principal afectación el bloqueo de la vía.

Palabras claves: Riesgo, Remoción en masa, Precipitación, Precipitación efectiva, Hidrología y Suelos.

Cómo citar:

Rodríguez Mejía, C., Bustos Castelblanco, D., Cadena Acevedo, A., \& Salazar Henao, D (2019) EVALUACIÓN DEL RIESGO POR MOVIMIENTOS DE REMOCIÓN EN MASA ACTIVADOS POR PROCESOS DE PRECIPITACIÓN, ESTUDIO DE CASO VÍA CHAGUALÁ - QUINDÍO. Revista de Investigaciones Universidad del Quindío. Vol 31, pp.7-14. 


\begin{abstract}
This document presents the risk assessment for mass removal movements activated by precipitation processes on the Chagualá road in the department of Quindío; for which it is taken as the alignment of the methodologies developed by the National Unit for Risk and Disaster Management (UNGRD) and the Colombian Geological Service, which consist of estimating the threat by means of the scientific analysis of the parameters hydroclimatic, geological, physical, geometric and mechanical aspects of the study area, as well as the estimation of the risk factor, through the simplified bishop method; the vulnerability determination is based on the impact matrices and by means of the information cruise the risk of slip by precipitation is evaluated. The results of the study showed that the value and variation of the rainfall intensity in the function of the return period is negligible, compared to the water sheet generated by the total annual precipitation, in addition to the risk analysis showed a high level before a movement of mass removal considering as main affectation the blockage of the road.
\end{abstract}

Keywords: Risk, Mass Removal, Precipitation, Effective Precipitation, Hydrology and Soils.

\section{INTRODUCCIÓN}

En Colombia se presentan emergencias sociales, económicas y ambientales producidas por movimientos en masa de laderas, las cuales pueden causar desplazamientos civiles junto con pérdidas de cultivos y viviendas; por lo tanto, es necesario aplicar métodos que permitan analizar el riesgo para estos eventos (IDEAM, 2012).

En la serie de estudios realizados alrededor del mundo se evidencia la interacción entre los fenómenos de precipitación y los desplazamientos de laderas en forma de flujos de lodo, derrumbes y/o desprendimientos (Corominas, 2006); y traen a colación la gran importancia que tienen las condiciones climáticas (Servicio Meteorológico Nacional de Estados Unidos, 2015) y la naturaleza del suelo en la producción de deslizamientos (Sánchez, 2011). Hay que resaltar que los taludes propensos a movimientos de remoción en masa activados por precipitación pueden ser tratados para la disminución de la amenaza (Huang, Ju, Liao, \& Liu, 2015), no obstante, estos procedimientos son sumamente costosos con resultados localizados y poco eficientes; con el fin de disminuir el impacto generado por estos movimientos, es posible y más beneficioso la reducción de la vulnerabilidad de los elementos expuestos y/o de la población que puede ser afectada (Organización Panamericana de la Salud).

Los movimientos de remoción en masa son even- tos recurrentes en la zona rural y urbana de Colombia (Aristizábal, Martínez, \& Vélez, 2010; DANE, 2013; IDEAM, 2000); en el departamento del Quindío, a pesar de ubicarse en pisos térmicos donde las precipitaciones son frecuentes, carece de procesos eficientes y efectivos para el registro de variables hidroclimatológicas con granularidad alta, así como las afecciones que las alteraciones de estas variables generan. Lo anterior, suscita la necesidad de la generación temprana de alarmas para las cuales se requiere del uso de herramientas e información de apoyo con la cual se puede realizar estudios o modelaciones que faciliten el conocimiento del evento amenazante, para este caso los movimientos de remoción en masa activados por precipitación. Esta recolección de información se facilita si se tiene un procedimiento base, de esta forma el análisis del riesgo por la desestabilización en taludes puede surgir como una amenaza estudiada disminuyendo el riesgo frente a ella (Aristizábal, Gamboa, \& Leoz, 2010). Razones por las cuales como estudio de caso se realiza la evaluación del riesgo de remoción en masa activado por procesos de precipitación en un talud ubicado sobre la vía Chagualá en el departamento del Quindío.

\section{METODOLOGÍA}

El desarrollo de la evaluación del riesgo de remoción en masa activado por procesos de precipitación se rige bajo el siguiente enfoque metodológico: 


\section{Zona de Estudio}

El departamento del Quindío está situado en el centro geográfico del occidente colombiano. En su división político-administrativa se integra con 12 municipios, entre los cuales se encuentra el municipio de Calarcá. El acceso más importante al municipio es a través de la ruta nacional 40; ruta que atraviesa el país de occidente a oriente, para conectar principalmente las ciudades de Buenaventura y Bogotá, siendo esencial para el transporte de mercancías. Con respecto al trazado de la ruta 40, entre Calarcá y Armenia, existe una vía principal (La María) y una variante (Chagualá); ambas poseen múltiples taludes artificiales en toda su extensión y en épocas lluviosas sufren de movimientos de remoción en masa.

La selección del sitio de estudio fue realizada por medio de inspección visual, para ello se recorrió toda la vía Chagualá y se ubicaron los taludes más representativos teniendo en cuenta la longitud, la altura y la cobertura del suelo, asimismo se asignó una mayor puntuación a los taludes que poseían obras de contención.

Con base en las características mencionadas, el talud más adecuado para estudiar fue el ubicado en $\mathrm{K} 4+300$ sentido Armenia-Calarcá, con coordenadas $\mathrm{X}=1159643$ y $\mathrm{Y}=995911$, este había sufrido un movimiento de remoción en masa hace pocas semanas y tenía señales de otro deslizamiento de mayor intensidad (ocurrido hace varios meses o años), además ya se encontraba intervenido con gaviones en la pata del talud y con un sistema de drenaje en concreto cerca de la corona. Por otra parte, las dimensiones del mismo eran considerables, el área superficial poseía escaza cobertura vegetal y su ubicación era muy cercana a la vía, por lo tanto, su zona de impacto era representativa.

\section{Caracterización del sitio de estudio}

El estudio de suelos del talud se realizó por medio de tres sondeos, extrayendo muestras alteradas por medio de bolsas y del Split Spoon: el primero se ubicó en la corona del talud y perteneció a un estrato arenoso; el segundo sondeo se elaboró en la pata del talud y su estrato predominante fue limo-arenoso; y el último sondeo fue una muestra en bloque ubicada a 1.5 metros sobre la pata.

Con las muestras en bolsa se determinaron los límites en donde el estrato presentaría un comportamiento plástico y líquido, utilizando el procedimiento denominado Límites de Atterberg, además se obtuvo la composición del suelo a partir de granulometría por tamizado y la humedad natural por medición volumétrica; con las extraídas por el Split Spoon se calcularon las densidades de cada uno de los estratos, de donde se calculó el peso específico del suelo (peso que ocupa un cuerpo por unidad de volumen). Dado que con la muestra de bloque se asegura resultados de mejor calidad, se determinaron de nuevo las variables mencionadas, así como la cohesión y el ángulo de fricción del suelo (variables que determinan la resistencia al desplazamiento de un suelo), por medio del ensayo del corte directo, datos fundamentales para estimar el factor de seguridad ante un deslizamiento en el talud.

Por otro lado, la modelación geométrica fue realizada por medio del levantamiento topográfico del talud y la vía adyacente, para ello se construyó una nube de puntos con la ayuda de una estación total; además del procesamiento en AUTOCAD Civil 3D se realizó el modelado tridimensional del cual se extrajeron tres perfiles del talud. El primero se ubicó en la mitad del plano de falla donde se evidenció un deslizamiento reciente; el segundo atravesó el Sondeo 2 (vegetación pobre) y el último se situó sobre un área con vegetación media. Cabe mencionar, que todos los perfiles poseían una estratificación homogénea, visible por la erosión del talud.

Los registros de precipitación diarios fueron suministrados por la Corporación Autónoma Regional del Quindío (CRQ), debido a que esta cuenta con la estación meteorológica más cercena a la zona de estudio. Una vez se obtuvo la serie histórica, se determinó una ventana de tiempo de 
23 años, comprendida entre los años del 1989 y 2011, puesto que en estos se presentó la menor cantidad de datos faltantes de lluvia diaria. Una vez escogido el periodo de estudio se procedió a determinar la precipitación máxima anual para posteriormente ser convertida en precipitación efectiva utilizando el método establecido por el Soil Conservation Service (SCS), posteriormente estos se sometieron al análisis basado en las metodologías establecidas para Colombia, propuestas por Díaz-Granados para la obtención de curvas Intensidad-Duración-Frecuencia (IDF) con base en datos pluviométricos. Teniendo en cuenta lo anterior y la guía Metodológica para estudios de amenaza, vulnerabilidad y riesgo por movimientos en masa del Servicio Geológico Colombiano (2015), se calculó la profundidad del nivel freático por medio de la siguiente ecuación:

$$
P_{f T R}=\left(\overline{P_{f}}-1,65 * C V * \bar{P}_{f}\right)-\left(I_{T R} * 24\right)
$$

Ecuación 1. Profundidad del nivel freático asociado al periodo del retorno TR

Donde:

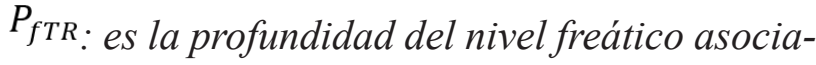
do al periodo del retorno TR.

$\bar{P}_{f}:$ corresponde a la profundidad inicial

$C V$ : es el coeficiente de variación

$I_{T R}$ :corresponde a la intensidad según el periodo de retorno

\section{Caracterización de la amenaza}

Una vez obtenida la geometría y las características físicas del suelo, así como la lámina de agua esperada; se procedió a determinar la superficie de falla y su respectivo factor de seguridad. Para ello, se utilizó un análisis de agua subterránea a partir de superficies de agua, además se implementó el método de Bishop simplificado (a través de equilibrio de fuerzas).

Lo anterior, se hace a través del software SLIDE; en este se graficó el perfil del suelo, se suministra- ron las características del suelo (peso específico, cohesión y ángulo de fricción), se seleccionaron los métodos de análisis mencionados y luego se graficó el nivel freático del talud (altura en donde se observó la lámina de agua). Una vez modelado el perfil, el programa calculó la superficie de falla y su respectivo factor de seguridad.

Con base en el modelado de cada uno de los taludes, se estimó la amenaza en función del área de material desplazado y el factor de seguridad predominante, estos datos son condensados en la matriz de amenaza y se promedian, agrupándose los perfiles iniciales y los calculados; se destaca, que la frecuencia no se tuvo en cuenta para determinar la amenaza, porque como se mencionó, la variación de la lámina de agua en función del periodo de retorno fue despreciable.

\section{Caracterización de la vulnerabilidad}

De acuerdo con la metodología planteada por la Unidad Nacional para la Gestión del Riesgo de Desastres (UNGRD) se realizó el estudio pertinente para la clasificación del nivel de vulnerabilidad que se presentó en la zona de estudio; puesto que el sitio no se encontraba poblado, la calificación socio-económica se tornó de difícil estimación, por ello se decidió hacer un paralelo entre los factores que intervinieron en la vulnerabilidad directa del lugar y en la población más cercana (municipio de Calarcá).

Para la caracterización de la vulnerabilidad se consideraron factores físicos, económicos, físicoespaciales, sociales y ambientales; cada uno de ellos con diferentes números de variables a tener en cuenta, los cuales fueron calificados con un valor de 1, 2 o 3, siendo 1 la calificación óptima y 3 el que muestra el peor escenario posible.

\section{Evaluación del riesgo}

Esta etapa se desarrolló con la misma metodología propuesta por la UNGRD, la cual fundamenta la evaluación del riesgo en una matriz de doble entrada: "Matriz Amenaza y Vulnerabilidad" 
(Tabla 1); a partir de esta, teniendo en cuenta la intersección de los valores promedio de amenaza y vulnerabilidad determinados, se calificó el nivel de riesgo del área de estudio.

Tabla 1. Matriz de Peligro y Vulnerabilidad para Estimación del nivel de riesgo

\begin{tabular}{|c|c|c|c|}
\hline $\begin{array}{c}\text { Amenaza } \\
\text { Alta }\end{array}$ & Riesgo Medio & Riesgo Alto & Riesgo Alto \\
\hline $\begin{array}{c}\text { Amenaza } \\
\text { Media }\end{array}$ & Riesgo Bajo & $\begin{array}{c}\text { Riesgo } \\
\text { Medio }\end{array}$ & Riesgo Alto \\
\hline $\begin{array}{c}\text { Amenaza } \\
\text { Baja }\end{array}$ & Riesgo Bajo & Riesgo Bajo & $\begin{array}{c}\text { Riesgo } \\
\text { Medio }\end{array}$ \\
\hline & $\begin{array}{c}\text { Vulnerabili- } \\
\text { dad Baja }\end{array}$ & $\begin{array}{c}\text { Vulnerabili- } \\
\text { dad Media }\end{array}$ & $\begin{array}{c}\text { Vulnerabi- } \\
\text { lidad Alta }\end{array}$ \\
\hline \multicolumn{4}{|c|}{ de Desastres, 2013) } \\
\hline
\end{tabular}

Para cada uno de los perfiles del talud se realizó el respectivo análisis de riesgo, discriminando entre el nivel freático presente (a) y el calculado (b); se obtuvieron seis posibles comportamientos. El nivel de riesgo resultante de cada perfil se promedió con sus semejantes (inicial y calculado), y así se determinó un nivel de riesgo para el talud completo.

\section{RESULTADOS}

\section{Características físicas y mecánicas del suelo}

Una vez realizado los ensayos para los diferentes sondeos, se obtuvieron las siguientes características físicas y mecánicas del suelo (Tabla 2):

Tabla 2. Características físicas y mecánicas del suelo

\begin{tabular}{|c|c|c|c|c|c|}
\hline Sondeo & $\begin{array}{c}\text { Humedad } \\
(\%)\end{array}$ & $\begin{array}{c}\text { Límite líquido } \\
(\%)\end{array}$ & $\begin{array}{c}\text { Límite plástico } \\
(\%)\end{array}$ & $\begin{array}{c}\text { Peso específico seco } \\
(\mathrm{KPa} / \mathrm{m})\end{array}$ & $\begin{array}{c}\text { Tipo de } \\
\text { suelo* }\end{array}$ \\
\hline 1 & 44,18 & 51,07 & 44,00 & 11,87 & SM \\
\hline 2 & 44,31 & 45,31 & 39,64 & 13,32 & SM-MS \\
\hline 3 & 34,84 & 48,00 & 36,32 & 14,25 & MS-SM \\
\hline
\end{tabular}

*SM significa arena limosa y MS denota limo arenoso.

La cohesión determinada para los perfiles es cercana a cero y el ángulo de fricción es igual a $40.2^{\circ}$.

\section{Análisis de nivel freático}

El análisis de los registros de precipitación permitió calcular la precipitación efectiva del suelo, no obstante, el estudio tuvo en cuenta el cálculo del nivel freático modelando de dos formas la precipitación efectiva, según la intensidad de esta lluvia y su variación total anual. Se observó que el incremento de la lámina de agua resultante del análisis de intensidad posee una variación entre los periodos de retorno de 2 años y de 100 años igual a $13 \mathrm{~cm}$, con un valor base de $13 \mathrm{~cm}$, que comparado con 11 metros resultantes por el coeficiente de variación y el nivel freático inicial, es depreciable (ver Tabla 3).

Tabla 3. Nivel freático en función del periodo de retorno

\begin{tabular}{|l|c|c|c|c|c|c|c|}
\hline Periodo de Retorno (años) & $\mathbf{2}$ & $\mathbf{5}$ & $\mathbf{1 0}$ & $\mathbf{2 0}$ & $\mathbf{2 5}$ & $\mathbf{5 0}$ & $\mathbf{1 0 0}$ \\
\hline Intensidad de lluvia Infiltrada $(\mathrm{mm} / \mathrm{h})$ & 5,51 & 6,50 & 7,37 & 8,35 & 8,69 & 9,84 & 11,15 \\
\hline Nivel Freático por Intensidad $(\mathrm{m})$ & 0,13 & 0,15 & 0,18 & 0,20 & 0,21 & 0,23 & 0,27 \\
\hline Nivel Freático calculado $(\mathrm{m})$ & 11,24 & 11,22 & 11,20 & 11,17 & 11,17 & 11,13 & 11,10 \\
\hline
\end{tabular}

Además, se tuvo en cuenta que la profundidad del nivel freático inicial fue medida para todos los perfiles a partir del cambio de estrato $(20 \mathrm{~m}$ de altura desde la pata), porque el suelo superior poseía un canal en concreto que evitaba la acumulación de lluvia. Así, se asume que este suelo solo proporcionará carga al modelo, ya que nunca va a poseer una lámina de agua. 
Teniendo en cuenta lo anterior se calculó la lámina de agua para cada uno de los perfiles, obteniendo los resultados de la Tabla 4.

Tabla 4. Características hidrológicas del talud

\begin{tabular}{|l|l|l|}
\hline Variable & Perfil 1 y 2 & Perfil 3 \\
\hline $\begin{array}{l}\text { Profundidad del nivel freá- } \\
\text { tico inicial (m) }\end{array}$ & 20,55 & 20,55 \\
\hline $\begin{array}{l}\text { Precipitación efectiva total } \\
\text { anual promedio }\end{array}$ & 615,64 & 967,16 \\
\hline Desviación estándar & 166,57 & 208,44 \\
\hline $\begin{array}{l}\text { Coeficiente de variación } \\
(\%)\end{array}$ & 27,06 & 21,55 \\
\hline CN (Grupo B) & 61 & 79 \\
\hline $\begin{array}{l}\text { Profundidad del nivel freá- } \\
\text { tico final (m) }\end{array}$ & 13 & 11,2 \\
\hline
\end{tabular}

\section{Caracterización de Amenaza}

A partir de los insumos descritos y la modelación en SLIDE se obtuvieron los siguientes perfiles:

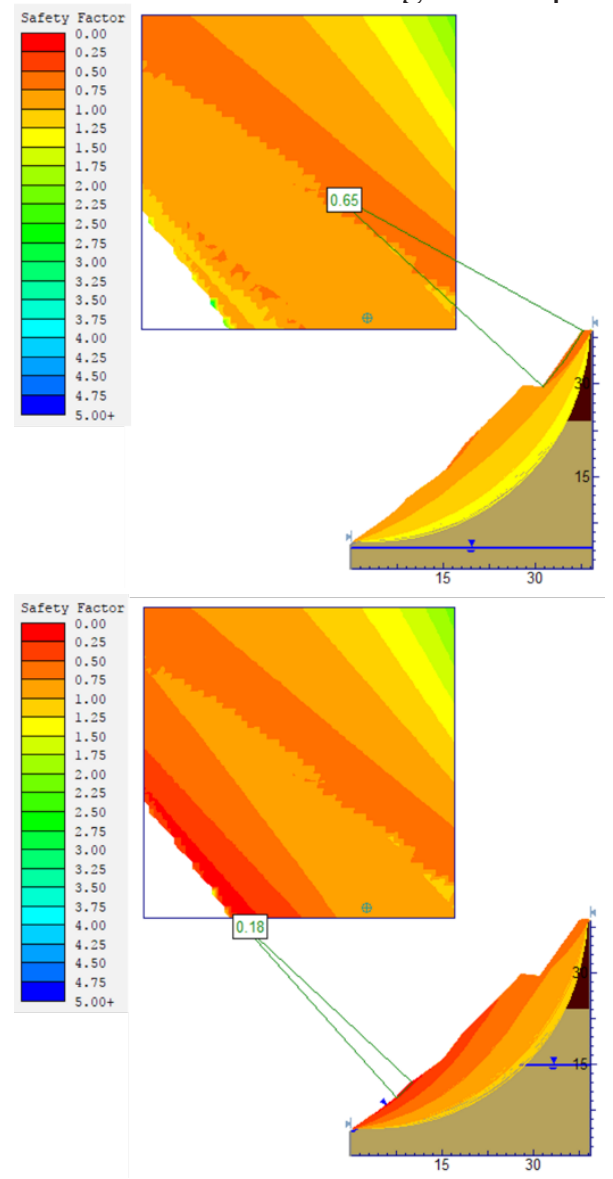

Imagen 1. Perfil 1: Ubicado sobre el sondeo 2 y el centro del talud
El Perfil 1 mostró que el área de deslizamiento posee un factor de seguridad de 2 o menos para el talud con nivel freático inicial (izquierda) y calculado (derecha); la zona naranja con mayor intensidad en el talud inicial presentó un factor igual o menor a 1, por tal motivo este presentó una tendencia a fallar, sin embargo, con la modelación calculada el factor decrece considerablemente y la totalidad de la zona de falla se encuentra por debajo de 1 en términos de seguridad.

Para el segundo perfil se tuvieron las mismas condiciones que el anterior, no obstante, la irregularidad en la superficie del terreno y el nivel freático más alto, considerando la altura total del perfil, disminuye el valor para el factor de seguridad. Sin embargo, ambos presentaron un grado de amenaza alto asociado a los movimientos de remoción en masa.

Aun cuando el comportamiento del perfil 3 es similar al de los demás perfiles, se debió considerar que la cobertura vegetal cambió el número de curva y por ende la profundidad del nivel freático; lo anterior se traduce en una disminución de la amenaza por el decrecimiento de la lámina de agua.

Con base en los modelos presentados, se caracterizó la amenaza con la siguiente matriz de análisis:

Tabla 5. Amenaza para cada perfil de estudio

\begin{tabular}{|c|c|c|}
\hline Amenaza & Valoración & Resultado \\
\hline Perfil 01-a & 1 & Baja \\
\hline Perfil 01 -b & 3 & Alta \\
\hline Perfil 02-a & 1 & Baja \\
\hline Perfil 02-b & 3 & Alta \\
\hline Perfil 03-a & 2 & Baja-Media \\
\hline Perfil 03-b & 3 & Alta \\
\hline
\end{tabular}

\section{Caracterización de la vulnerabilidad}

Según la valoración de cada uno de los aspectos se determinó el valor total, su promedio dio como resultado la valoración de la vulnerabilidad. En 
la Tabla 6, se muestra la recopilación del número de variables que se tienen en cuenta en cada caso y su respectiva calificación y clasificación final.

Tabla 6. Vulnerabilidad

\begin{tabular}{|l|c|c|}
\hline $\begin{array}{c}\text { Vulnerabilidad } \\
\text { Municipal }\end{array}$ & $\begin{array}{c}\text { Valoración } \\
\text { local }\end{array}$ & $\begin{array}{c}\text { Valoración } \\
\text { Municipal }\end{array}$ \\
\hline Económica & 0 & 7 \\
\hline Física & 9 & 11 \\
\hline Física-Espacial & 3 & 2 \\
\hline Social & 0 & 9 \\
\hline Ambiental & 7 & 8 \\
\hline Total & 2 & 2 \\
\hline \multicolumn{2}{|c|}{ Nivel Medio de Vulnerabilidad } \\
\hline
\end{tabular}

Como se pudo observar, el nivel de vulnerabilidad tanto en el sitio como en el municipio de Calarcá es medio, indicando que ambos escenarios son susceptibles a la ocurrencia de cualquier evento nefasto, por tanto, podrían verse perjudicados en el desarrollo normal y adecuado de sus respectivas actividades.

La diferencia más importante entre ambas valoraciones es la consideración de las afectaciones sociales, ya que la vulnerabilidad local solo considera los aspectos físicos (la vía), en cambio, la vulnerabilidad global considera también a la población, las entidades y sus relaciones.

\section{Caracterización del riesgo}

Del análisis del escenario actual se observa que la media de los tres perfiles poseen un nivel de riesgo bajo-medio, lo que quiere decir que el impacto y probabilidad actual generado por el deslizamiento es bajo; sin embargo, el análisis con el nivel freático calculado, muestra que el nivel de riesgo se incrementa y pasa a ser medio-alto o alto, lo que lleva a estimar que existe una alta probabilidad de deslizamiento de masa en un futuro próximo, si el comportamiento de la precipitación continúa con la misma tendencia.

\section{DISCUSIÓN Y CONCLUSIONES}

Con la caracterización del talud se logró determinar que el estrato predominante es limo-arenoso, de origen volcánico, el cual tiende a presentar fenómenos de inestabilidad debido a la humedad cercana al estado plastico y la baja cohesión; también, se encontró que el nivel freático está ubicado superficialmente, porque en el sondeo 2 se encontró a 0,55 $\mathrm{m}$ de profundidad desde la pata del talud.

Además, el estudio mostró que la variación del nivel freático en función del periodo de retorno es despreciable, ya que la profundidad inicial de la lámina de agua y el coeficiente de varianza de la precipitación efectiva total anual generan un nivel considerablemente mayor que el suministrado por la intensidad de la lluvia infiltrada diaria.

Según los resultados mostrados, la ocurrencia de un movimiento de remoción en masa activado por precipitación es probable y atiende a un nivel de riesgo medio-alto. Teniendo en cuenta que la estratigrafía de la zona es análoga a la estudiada, en cualquier punto de la vía pueden ocurrir las siguientes consecuencias: de forma directa, la vía quedaría obstaculizada, generando riesgo de pérdidas humanas debido al cruce del movimiento con vehículos o peatones. Por otra parte, las pérdidas económicas, físicas y ambientales están asociadas al desequilibrio en el desarrollo normal de las actividades del municipio de Calarcá, porque se limita la entrada al mismo y por consiguiente incurre en demoras de transporte, colapso vial y posiblemente desabastecimiento para los ciudadanos. Cabe mencionar, que esta vía aun cuando es una variante de la ruta 40, tiene registro de deslizamientos en fechas iguales a la de la vía principal, es decir, existen registros del cierre total de la ruta 40 por movimientos de remoción en masa asociados a la precipitación.

Se recomienda ampliar el área de estudio y aumentar la densidad de los sondeos para determinar las características y la estratificación del suelo, esto con el fin de presentar un modelo más preciso. 


\section{REFERENCIAS}

1. Aristizábal, E., Gamboa, M., \& Leoz, F. (julio de 2010). Sistema de Alerta Temprana por Movimientos en Masa Inducidos por Lluvia para el valle de Aburrá, Colombia. Revista EIA, 1237 (13), 155-169. Medellín, Colombia. Recuperado el 3 de noviembre de 2016, de [Base de Datos]: http://bit.ly/2eLWB31

2. Aristizábal, E., Martínez, H., \& Vélez, J. (2010). Una Revisión sobre el Estudio de Movimientos en Masa Detonados por Lluvias. Revista de la Academia Nacional de Ciencias, 34 (131), 209-227. Colombia.

3. Corominas, J. (2006). El Clima y sus Consecuencias sobre la Actividad de los Movimientos de Ladera en España. Recuperado el 16 de noviembre de 2016, de [Documento de Sitio Web]: http://bit. $\operatorname{ly} / 2 \mathrm{~g} 30 \mathrm{eEu}$

4. DANE. (2013). Número Registrado de Deslizamientos 2012. Recuperado el 9 de noviembre de 2016, de [Documento Digital]: http://bit.ly/2eqeEk6

5. Huang, J., Ju, N., Liao, Y., \& Liu, D. (21 de diciembre de 2015). Determination of Rainfall Thresholds for Shallow Landslides by a Probabilistic and Empirical Method. Natural Hazards and Earth System Sciences Discuss, 15 , 2715-2723. China. Recuperado el 18 de noviembre de 2016, de [Documento de Sitio Web]: http://bit.ly/2gbTjsD

6. IDEAM. (2000). Movimientos en Masa Dañinos Ocurridos en Colombia Durante el Fenómeno Frío del Pacifico ( La Niña) 1999 - 2000. Recuperado el 8 de noviembre de 2016, de [Documento Digital]: http://bit.ly/2flwSot

7. IDEAM. (2012). Metodología para la Zonificación de Susceptibilidad General del Terreno a los Movimientos en Masa. Recuperado el 8 de noviembre de 2016, de [Documento de Sitio Web]: http://bit. $1 \mathrm{y} / 2 \mathrm{fL} 9 \mathrm{Qnb}$

8. Organización Panamericana de la Salud. (s.f.). Disaster-info. Recuperado el 25 de noviembre de 2016, de [Documento de Sitio Web]: http://www.disaster-info.net/watermitigation/e/publicaciones/EstudioVEN/cap3.PDF

9. Sánchez, D. (6 de julio de 2011). Precipitaciones Extremas y sus Implicaciones en Procesos de Remoción en Masa en la Planificación Urbana de Tampico, México. BIBLID, 48, 135-159. México. Recuperado el 19 de noviembre de 2016, de [Documento de Sitio Web]: http://bit.ly/2g9C3Xi

10. Servicio Meteorológico Nacional de Estados Unidos. (4 de noviembre de 2015). Cold and Warm Episodes by Season. Recuperado el 9 de noviembre de 2016, de [Sitio Web]: http://bit.ly/1NCHILt

11. Unidad Nacional para la Gestión del Riesgo de Desastres. (2013). Guía Metodológica para la Elaboración de Planes Departamentales para la Gestión del Riesgo. Recuperado el 18 de noviembre de 2016, de [Documento de Sitio Web]: http://bit.ly/2fPDkC5 